\title{
Indentità nazionale e immagini dell'“altro" nella narrativa di Pier Antonio Quarantotti Gambini
}

\author{
Lara Sorgo \\ Università del Litorale, Facoltà di Studi Umanistici
}

Pier Antonio Quarantotti Gambini, pisatelj iz plemiške, iredentističnim idealom predane družine, je vedno poudarjal italijanskost Istre in Julijske krajine. V njegovih pripovednih delih, zlasti romanih, ki sestavljajo zbirko Gli anni ciechi (197I), se italijanska nacionalna identiteta izraža tako skozi jezik kot kulturo.V pričujočem prispevku smo kritično preučili literarne podobe, ki jih posreduje besedilo, samopredstavitve (auto-images) in podobe $\gg$ drugega $\ll$ (hetero-images) ter poudarili vlogo literature pri ustvarjanju nacionalnih mitov, ki napajajo specifično domišljijo.

Ključne besede: nacionalna identiteta, drugačnost, imagologija, literatura, Pier Antonio Quarantotti Gambini

Pier Antonio Quarantotti Gambini, a writer from an aristocratic family of irredentist ideals, has always highlighted the Italianness of Istria and Venezia Giulia. In his narrative works, especially in the novels of the collection Gli anni ciechi (197I), the Italian national identity is expressed through language and culture. In this contribution the literary images transmitted by the novels, the self-representations (auto-images) and the representation of the Other (hetero-images), were critically analyzed, highlighting the role of literature in the construction of national myths that feed specific imaginaries.

Key words: national identity, otherness, imagology, literature, Pier Antonio Quarantotti Gambini

$\mathrm{P}$

ier Antonio Quarantotti Gambini in una nota del 1947 afferma:

devo constatare amaramente che vengo considerato, come centinaia di miei conterranei altrettanto italiani di me, alla stregua di uno che non si sa più che cosa sia: uno che ha dinanzi a sé tre possibili cittadinanze, due con strascichi, complicazioni, incompatibilità, e una addirittura straniera. Come un uomo, insomma, che gli italiani reputano italiano, i triestini triestino e gli jugoslavi cittadino della federazione di Tito (Moretto e Picamus 2010, 29).
Una tale considerazione dimostra l'interesse di Quarantotti Gambini per la natura stessa dell'identità che, di volta in volta, può essere plasmata da un comune passato storico, dal luogo in cui si è vissuti, dalla lingua che si parla, dalle tradizioni e dalle convenzioni sociali tramandate, ma anche dall'esperienza personale individuale.

La vita dell'autore infatti si intreccia ai grandi sconvolgimenti politici della prima metà del XX secolo. Nato a Pisino d'Istria nel igro al tempo dell'Impero asburgico, nel I9I8 visse il momento del passaggio dei territori al Regno d'Italia e molti anni dopo nel 1945, è stato testimone dell'occupazione di Trieste da parte dell'eserci- 
to di Tito e del passaggio dell'Istria, del Quarnero e della Dalmazia ai territori della Jugoslavia.

Nell'area geografica che fa da sfondo alla vicenda personale dell'autore, e che compare anche nei suoi romanzi, l'identità etnica e l'identità nazionale spesso si sovrappongono e vengono usate come sinonimo l'una dell'altra. La sua esperienza personale permette una prima spiegazione agli esempi di auto-rappresentazioni ed etero-rappresentazioni che si possono ritrovare nelle opere narrative. Infatti è soprattutto durante i periodi di instabilità politica o segnati da conflitti bellici, che assumono grande importanza le rappresentazioni del "sé", sia in quanto singoli individui sia come membri di una collettività, contrapposti ai cosiddetti “altri”, singole persone o una collettività come la nazione.

\section{L'imagologia letteraria}

Nonostante l'interesse principale della letteratura comparata è dato dall' incontro con i testi letterari stranieri e con le culture diverse dalla nostra $^{1}$, la prospettiva imagologica, specialismo di tale ambito, sarà l'approccio privilegiato da usare per la seguente analisi. Secondo la definizione di Nora Moll, l'imagologia

è un campo di ricerca della comparatistica
letteraria incentrato sullo studio delle im-
magini del così detto 'altro' o 'straniero', at-
traverso le quali è possibile risalire alle strut-
ture mentali individuali e collettive atte a
formulare definizioni e giudizi più o meno
costanti su chi è estraneo rispetto alla nazi-
one, alla cultura o al gruppo etnico d'appart-
enenza (Moll 2018,157$)$.

Dal punto di vista dell'imagologia quindi, la letteratura ricopre un ruolo centrale

$$
\begin{aligned}
& \text { all'interno del secolare processo di recip- } \\
& \text { roche definizioni e visioni, un ruolo di catal- } \\
& \text { izzatore di idee, immagini e stereotipi, ma } \\
& \text { anche di incunabolo di nuove visioni e di } \\
& \text { scenari ignoti (Moll 20I8, } 158 \text { ). }
\end{aligned}
$$

Per il francese Yves Chevrel, "il punto di partenza e l'interesse essenziale della letteratura comparata è dato dall'incontro con l'"'altro"' (Moll 1999, 211).
Allo stesso tempo però, l'analisi critica delle immagini letterarie di un'altra cultura si intrecciano con la visione che un autore ha della propria cultura, quindi con quella che possiamo definire l'identità culturale, "attraverso un continuo confronto che muove dall'identità all'alterità, giacché parlare degli "altri" è sempre anche un modo per rivelare qualcosa di sé" (Moll 1999, 213).

Gli aspetti più recenti della disciplina si riconoscono nell'opera di Manfred Beller e Joep Leerseen. Partendo dalla considerazione che esistono diverse tipologie di immagini, e che nel campo dell'imagologia con il termine immagine si indica "the mental silhouette of the other," (Beller e Leerssen 2007, 4), le differenze culturali percepite possono guidare giudizi e opinioni sugli altri e anche il modo in cui vengono codificati simili comportamenti. Lo scopo dell'imagologia, come viene descritto dai due studiosi, è "to describe the origin, process and function of national prejudices and stereotypes, to bring them to the surface, analyse them and make people rationally aware of them"3 (Beller e Leerssen 2007, II-I2). Compito dell'imagologia è quindi quello di riconoscere un discorso di rappresentazione negli autori che saranno analizzati, senza però escludere la soggettività delle fonti impiegate, che anzi deve essere tenuta in considerazione. Importanti saranno quindi non solo le immagini dell'“altro" (hetero-images), ma anche le immagini del "sé" (self-images, auto-images), ovvero quelle caratterizzazioni e quei attributi astratti e stereotipati che Leerssen definisce "immaginate" (imaginated).

Queste premesse metodologiche offrono degli spunti di riflessione interessanti per condurre l'analisi critica delle opere letterarie di Pier Antonio Quarantotti Gambini. Innanzitutto il fatto che l'interpretazione delle images letterarie riguarderà l'analisi letteraria e non un'analisi storica o sociologica, quindi si individuerà un

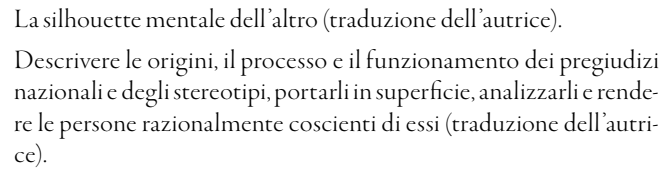
nazionali e degli stereotipi, portarli in superficie, analizzarli e rendere le persone razionalmente coscienti di essi (traduzione dell'autrice) 
discorso di rappresentazione letteraria e non una rappresentazione della società; inoltre, la prospettiva imagologica può risultare riduttiva per poter avere un quadro completo della produzione letteraria che si intende analizzare. A questo scopo, si dovrà quindi ricorrere anche alla critica tematica, che permetterà di individuare alcune tematiche ricorrenti nei libri analizzati, e ai metodi propri della narratologia, utili per un'analisi approfondita dei testi. Ogni volta quindi il discorso critico di analisi sarà portato avanti scegliendo gli strumenti che meglio di altri si adattano ad estrapolare dai testi gli aspetti e i significati che si vogliono studiare.

\section{Pier Antonio Quarantotti Gambini: vita e opere}

Pier Antonio Quarantotti Gambini nacque a Pisino d'Istria nel 1910 da Giovanni Quarantotto, che apparteneva a un'antica famiglia nobile di Rovigno e da Fides Histriae Gambini, discendente di un'illustre famiglia capodistrianas. Presso la villa del nonno materno a Semedella, sul litorale vicino a Capodistria, passò gran parte dell'infanzia e dell'adolescenza, e proprio a questi luoghi si deve la sua ispirazione letteraria. Durante gli anni di studio presso il liceo Carlo Combi a Capodistria, incontrò lo scrittore inglese Richard Hughes, a Trieste conobbe Umberto Saba con il quale strinse un'amicizia duratura. Questi incontri furono decisivi per il giovane Quarantotti Gambini perché lo incoraggiarono nelle sue prime prove di scrittore (Zudič Antonič 2014, 400-40I; Iannuzzi 2016).

Iniziati gli studi di giurisprudenza all'Università di Milano, li portò a termine nel 1937 all'Università di Torino. Nel frattempo, tra il I929 e il 1932, esordì come narratore sulla rivista Solaria con le tre novelle I tre crocifissi, Il fante di spade e La casa del melograno che, per interessa-

4 Il nonno paterno era discendente di una famiglia nobile di Rovigno, quella dei Quarantotto. Pier Antonio, fece modificare il cognome nel 1937 (Zudič Antonič 2014, 400).

5 Il nonno materno, da cui Pier Antonio prese il nome e il cognome, era stato un politico di rilievo nell'Ottocento: capo della maggioranza italiana alla Dieta provinciale di Parenzo, deputato parlamentare a Vienna e membro delle Delegazioni dell' Impero austro-ungarico (Iannuzzi 2016). mento di Umberto Saba e di Eugenio Montale, vennero raccolte e riunite nel 1932 con il titolo di Inostri simili (Zudič Antonič 2014, 402).

A partire dagli anni Trenta, Quarantotti Gambini collaborò a diverse riviste e testate italiane: L'Italia letteraria (1929), La Stampa (1933), nonché nel 1935 venne pubblicato a puntate sulla rivista Pan il primo romanzo, La rosa rossa, poi edito in volume nel 1937 per Treves. Tra gli anni Quaranta e Cinquanta, pubblicò anche su Il Tempo di Roma, su Il Tempo di Milano, in quotidiani triestini e riviste. Trovato impiego presso la Società di navigazione genovese Italia, ebbe modo di compiere numerosi viaggi (Iannuzzi 2016).

Nel 1939 aveva già finito la stesura del racconto Tre bandiere che dapprima fu pubblicato nel 194I nella rivista fiorentina Letteratura, quindi nel 1942 in volume nella collana "Narratori contemporanei” dell'editore Einaudi (Moretto e Picamus 2010, 27).

Nel 1942 il Municipio di Trieste gli affidò la direzione della Biblioteca civica Hortis e del suo Archivio diplomatico. A questo periodo risale il romanzo L'onda dell' incrociatore, pubblicato da Einaudi nel 1947 che l'anno successivo vinse il Premio Bagutta.

L'occupazione di Trieste nel maggio $1945 \mathrm{da}$ parte delle truppe dell'esercito jugoslavo di Tito, segnò drammaticamente la vita dello scrittore. Rifiutatosi di aderire alle associazioni titine che chiedevano l'annessione dell'Istria alla Jugoslavia, rischiò l'arresto e venne destituito dall'incarico di direttore della Biblioteca civica. Quarantotti Gambini scappò clandestinamente da Trieste per recarsi prima ad Udine e in seguito a Venezia. I ricordi dei quaranta giorni dell'occupazione titina di Trieste furono descritti in Primavera a Trieste. Ricordi del '45, uscito nel I95I per Mondadori (Moretto e Picamus 2010).

Tra il 1945 e il 1949 diresse l'emittente radiofonica clandestina Radio Venezia Giulia, in seguito si dedicò completamente alla scrittura. Negli anni Cinquanta uscirono Amor militare (prima edizione del 1955, successivamente pubblicato con il titolo L'amore di Lupo, 1964), Il cavallo Tripoli (1956) che andranno a costituire 
i romanzi del cosiddetto 'ciclo di Paolo' raccolti successivamente nella raccolta Gli anni ciechi ${ }^{6}$.

Nel 1958 uscì il romanzo La calda vita, opera esterna agli "anni ciechi" e tra le più ambiziose e controverse, mentre nel 1963 fu pubblicata l'opera di carattere diacritico e memorialistico, Sotto il cielo di Russia. Nel 1964, anno in cui all'autore fu affidato l'incarico di presidente dell'Associazione degli scrittori veneti, fu pubblicato il romanzo I giochi di Norma (Iannuzzi 2016).

Quarantotti Gambini mori prematuramente a Venezia nel 1965 per arresto cardiaco.

Gli scritti dell'autore rimasero al fratello Alvise che diede alle stampe i volumi di poesie $R a c$ conto d'amore (1965) e Al sole e al vento (1970), oltre alla già menzionata raccolta Gli anni ciechi (I97I) (Iannuzzi 2016).

\section{Identità nazionale e immagini dell'“altro"}

Ad una prima lettura, la maggior parte delle opere narrative di Pier Antonio Quarantotti Gambini sono percorse dalla coscienza di un'identità nazionale italiana a cui si contrappongono, di volta in volta, soldati austriaci, soldati italiani delle diverse regioni d'Italia e slavi.

Già nell'opera d'esordio dello scrittore, la raccolta di racconti $I$ nostri simili, la differenza tra gli italiani e gli 'altri' voleva dire possedere alcuni tratti somatici e alcune caratteristiche comportamentali, che gli istriani sentivano di avere in comune con gli italiani, ai quali si sentono profondamente legati per storia, tradizione e lingua (Gallo 2012, 1545).

Nella seconda opera di Quarantotti Gambini, La rosa rossa, diventa invece molto importante la questione della lingua, che viene sentita come fattore dell'espressione dell'identità nazionale e dell'appartenenza. Le vicende, ambientate a Capodistria nel periodo del primo dopoguerra, narrano il ritorno a casa del vecchio conte Paolo, ex generale dell'esercito austriaco. Il suo soggior-

6 La raccolta Gli anni ciechi uscì nel 1971 nella collana "Supercoralli" della Einaudiad opera del fratello Alvise Quarantotti Gambini che si preoccupò di curare l'edizione seguendo il progetto com'era stato concepito dal fratello (Qurantotti Gambini 1971, 657-659). Oltre ai romanzi che erano stati precedentemente pubblicati, alcuni vennero pubblicati postumi, mentre il breve racconto Le estati di fuoco fu pubblicato per la prima volta proprio in questa raccolta. no nella casa del cugino e della moglie, seppure molto breve perché il conte morirà di lì a poco, porta alla memoria vecchi ricordi che il protagonista "alterna alle considerazioni sulla fine dell'Impero austro-ungarico e sulla decadenza della nobiltà capodistriana" (Zudič Antonič 2007, 42). La lingua e la discendenza familiare quindi sono per Quarantotti Gambini due fattori importanti dell'espressione dell'identità nazionale (Gallo 2012, I546). Nei passi del libro leggiamo infatti che il conte Paolo

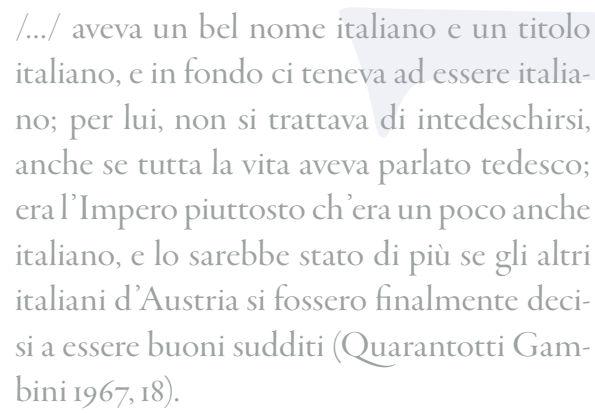

Che l'Impero austro-ungarico avesse una componente italiana, viene messa in evidenza da Quarantotti Gambini perché nella stessa pagina dice che l'Imperatore, "gli aveva rivolto la parola in toscano" (Quarantotti Gambini 1967, 18), a sottolineare di nuovo l'importanza della lingua nel suo stretto rapporto con l'appartenenza. Tuttavia, durante la prima guerra mondiale, il conte Paolo era stato guardato con sospetto per "il suo nome, le sue parentele, le sue simpatie con i popoli latini” (Quarantotti Gambini 1967, 84), mentre dopo il suo ritorno a Capodistria, suo cugino Piero temeva che l'ex generale austriaco potesse venir affrontato in pubblico da chi invece aveva combattuto in guerra sul fronte italiano (Gallo 2012, 1546).

Nelle ultime pagine del libro si fa riferimento invece alla situazione politico-sociale attuale. La signora Ines lamenta che "coi partiti non si capisce più niente, anche i nomi sono nuovi” (Quarantotti Gambini 1967, 257), a cui risponde suo marito Piero dicendo che sono solo i nomi ad essere nuovi, "ma la gente è sempre la stessa" (Quarantotti Gambini 1967, 257). 
È però soprattutto nel cosiddetto "ciclo di Paolo", serie di romanzi riuniti nella raccolta $G l i$ anni ciechi (197I), che la questione dell'identità nazionale è rinforzata $\mathrm{da}$ immagini relative $\mathrm{a}$ questioni identitarie che si inseriscono nei difficili rapporti tra diversi gruppi nazionali e/o culturali. Nel volume è possibile individuare una vera e propria linea di demarcazione tra "noi" e "loro", quindi tra Paolo (alter ego dello scrittore) e la sua famiglia, animati da un profondo spirito nazionale italiano, e gli altri. La raccolta è composta dai romanzi Le redini bianche, La corsa di Falco, Il cavallo Tripoli, L'amore di Lupo, Le estati di fuoco, I giochi di Norma.

Il ciclo si apre con il brano Tre bandiere, che compare all'inizio de Le redini bianche e fa da prologo all'intera struttura narrativa, e narra il ritorno da esule alla terra natale di Paolo de Brionesi Amidei, quindici anni dopo la fine del secondo conflitto mondiale. Il protagonista arriva a Capodistria a bordo di un panfilo partito da Venezia per una crociera estiva. I territori dell'Istria ora si trovano sotto il dominio della Repubblica federativa di Jugoslavia, anche se Paolo si rifiuta di considerare tali terre jugoslave:

Per la Jugoslavia? Per l'Istria! Perché /.../ egli
evitava di considerare come jugoslava l’Istria,
sebbene essa fosse ormai staccata dall'Italia:
tanto che bisognava entrarvi con tutti i con-
trolli e con tutte leformalità di quando si va
all'estero (Quarantotti Gambini 2o II, II).

Paolo, sebbene nato in quei luoghi ("Io sono di qui. Questi sono i paesi miei”; Quarantotti Gambini 20II, I5), si ritrova in un "mondo nuovo", di gente che parla un'altra lingua (“/.../ sentir parlare nella propria città, da tutti, una lingua che non si conosce!"; Quarantotti Gambini 20II, 27).

Il ricordo del passato serve a Paolo per parlare di avvenimenti storici drammatici che sono seguiti alla seconda guerra mondiale, quali le violenze jugoslave nei confronti della popolazione italiana dell'Istria, l'esodo, le foibe:

\footnotetext{
Tito è riuscito nel suo intento, e come! /.

Avevo sentito dire, è vero, e avevo anche let-

to sui giornali, che da Capodistria, da Isola
}

e da Pirano, dopo il '54 quasi tutti sono andati via, come da Pola nel '46... (Quarantotti Gambini 20II, 22).

Le terre che erano state lasciate dalle persone che se n'erano andate via, come le proprietà della famiglia di Paolo, sono state poi prese dai nuovi venuti:

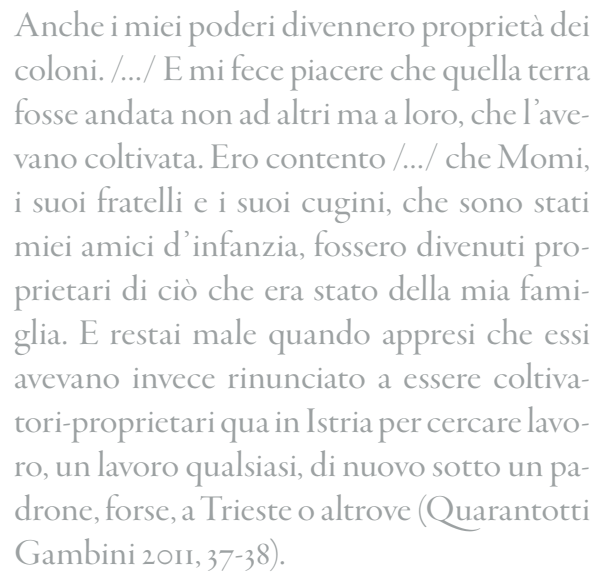

Con l'esodo che ha coinvolto anche i contadini delle città costiere, erano venuti ad abitare le cittadine istriane gli slavi mandati da Tito dai paesi al di là delle Alpi Giulie (Quarantotti Gambini 20II, 39).

Il brano si chiude con la riflessione di Paolo sulle tre differenti bandiere, da cui prende il titolo il racconto, che sono state issate a Capodistria nel corso di venticinque anni:

\section{La gialla e nera, imperiale, dell'Austria, anzi degli Asburgo, sino al I9r8, poi l'italiana, e poi, già nell'autunno del '43, quando fui qui una delle ultime volte, questa che sventola oggi: la bandiera jugoslava con la stella co- munista (Quarantotti Gambini 2oII, 44).}

I romanzi successivi riportano invece il lettore all'infanzia di Paolo passata a Semedella, nella villa del nonno, e a Trieste. Ad ogni romanzo fanno da sfondo vicende storiche e personaggi facilmente riconoscibili che si intrecciano con la storia personale della famiglia di Paolo. Così ne Le redini bianche, La corsa di Falco e Il cavallo Tripoli si attende lo sbarco degli italiani per libe- 
rare le terre istriane dalla dominazione austriaca; ne L'amore di Lupo, Le estati di fuoco e I giochi di Norma invece, si narra dell'età post-bellica.

$\mathrm{Ne}$ Le redini bianche siamo alla vigilia della prima guerra mondiale. Paolo ha quattro anni e trascorre la sua infanzia nella villa dei nonni a Semedella. Nel romanzo vengono descritte le esperienze che il bambino compie scoprendo il mondo attorno a sé e ascoltando i discorsi degli adulti. L'italianità dell'Istria viene costantemente ricordata e sperata nell'attesa di poter issare la bandiera italiana:

\begin{abstract}
La nostra bandiera /.../ è bianca rossa e verde: del colore degli oleandri e delle foglie che hanno intorno. Ma, sinché c'è l'Austria, non siamo "liberi" e non possiamo alzarla (Quarantotti Gambini 2011, 83).
\end{abstract}

Le popolazioni italiane dell'Istria inoltre attendono l'arrivo degli italiani per essere liberati dalla dominazione austriaca:

\section{Un giorno gli italiani sarebbero arrivati con \\ le navi, e sarebbe stato il più bel giorno della loro vita (Quarantotti Gambini 2011, 83).}

Il romanzo successivo, La corsa di Falco, narra di un imminente cambiamento politico-sociale, dovuto all'attentato dell'arciduca Francesco Ferdinando, erede al trono d'Austria-Ungheria, che segnerà lo scoppio della prima guerra mondiale. In questo clima si inseriscono i comportamenti della famiglia di Paolo, che dimostra la forte italianità: il padre infatti legge il Corriere delle Sera, la madre canta l'inno italiano, il nonno e lo zio Manlio discutono di questioni politiche. La stessa Italia, definita semplicemente dalla locuzione avverbiale "di là", assume i contorni di un luogo mitico dove ci si può aspettare di tutto, anche il pane bianco che ormai non si trova da tempo (Gallo 2012, I547).

I discorsi degli adulti che Paolo sente sono però difficili da comprendere per un bambino. Egli infatti sente nelle conversazioni a casa, parole come "offensiva", "avanzata", "ritirata", "alleati”, "neutralità dell'Italia”, "interventisti”, “triplice alleanza”, e gli viene raccomandato di non parlarne con nessuno, soprattutto di non far- ne parola con i servi che sono slavi (Gallo 2012, I547). La confusione di Paolo è tale da iniziare a porsi le prime domande sull'identità e a chiedersi:

$$
\begin{aligned}
& \text { Chi erano gli austriacanti, e chi gli slavi, di } \\
& \text { cui sentiva sempre parlare? /.../ Degli slavi, } \\
& \text { degli slavi e ancora degli slavi: Paolo lo sen- } \\
& \text { tiva ripetere continuamente questa parola } \\
& \text { (Quarantotti Gambini 1969, I26-I27). }
\end{aligned}
$$

Ne Il cavallo Tripoli, che descrive l'occupazione della villa della famiglia Amidei da parte di un ufficiale austriaco, i soldati austriaci sono considerati nemici con cui non si deve parlare, ma per cui tuttavia Paolo prova simpatia. Il soldato Hans infatti, parla con lui italiano, e ciò è un fatto importante perché vuol dire riconoscere nella lingua il segno distintivo di un popolo (Gallo 20I2, I548). Hans inoltre, racconta a Paolo che aveva conosciuto i soldati italiani e li giudica tutt'uno con quelli austriaci:

$$
\begin{aligned}
& \text { Egli ne parlava /.../ non come di nemi- } \\
& \text { ci ma come di camerati; come di camerati } \\
& \text { che combattessero, chi sa perché, dall'altra } \\
& \text { parte; quasi che austriaci o italiani fossero } \\
& \text { tutt uno: soldati, e nient'altro; soldati mes- } \\
& \text { si a combattere gli uni di qua e gli altri di là } \\
& \text { (Quarantotti Gambini } 1963,16 \text { ). }
\end{aligned}
$$

Paolo, dopo aver ascoltato le parole di Hans e durante una conversazione avuta con la madre, continua a porsi domande sulla questione della nazionalità e delle diverse ideologie politiche. Paolo scoprirà che non sono soltanto loro, i padroni, ad essere italiani, ma che lo sono anche $\mathrm{i}$ contadini, e che tra contadini italiani e contadini slavi non vi è alcuna differenza di valore (Quarantotti Gambini 1963, 23-24; Maculotti 2016, 73):

$$
\begin{aligned}
& \text { - Non sono slavi, i contadini? Perché non } \\
& \text { sono slavi? Italiani siamo noi! - Ce ne sono } \\
& \text { anche di slavi, ma stanno più lontano, non } \\
& \text { qui a Capodistria. (Quarantotti Gambini } \\
& \text { 1963,24). }
\end{aligned}
$$

In alcune occasioni i personaggi esprimono dubbi sulla propria o sull'altrui identità nazio- 
nale. Nella stessa opera, si individua un confronto tra due personaggi che sono caratterizzati da due opposte posizioni ideologiche e che confermano l'importanza della lingua: l'avvocato Tomaso, fratello del capitano austriaco, è italiano e assieme alla cognata preferisce parlare francese, perché lui non vuole parlare tedesco e lei non vuole parlare italiano: "Tedesco non voglio parlare io, italiano non vuole lei /.../ Il francese le va! Come se i francesi non fossero nemici dell'Austria altrettanto di noi..." (Quarantotti Gambini 1963,54$)$.

Il romanzo termina con la disfatta definitiva dell'Austria e la vittoria dell'Italia:

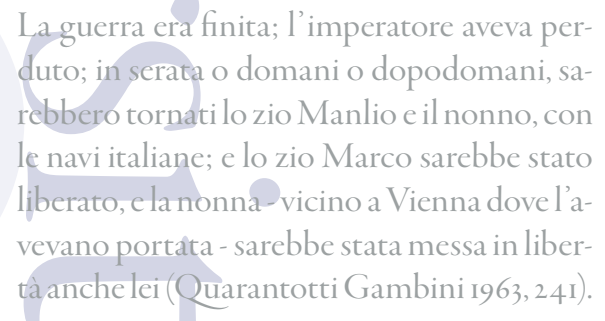

Gli italiani allora sono descritti come "gente perbene, la gente più civile del mondo, coraggiosi, gentili e leali" (Quarantotti Gambini 1963, 196).

Un altro caso in cui Paolo prova difficoltà nel capire che cosa sia l'appartenenza nazionale riguarda il caso dell'ufficiale Giusì che viene descritto nel racconto Le estati di fuoco. I fatti si rifanno al periodo postbellico. Paolo si ritrova a Semedella durante l'estate e in questo periodo conosce i figli di Giusì. Giusì, amico dello zio Manlio e giustiziato in guerra dagli austriaci perché "/../ era scappato "di là" per arruolarsi nella marina italiana e combattere contro l'Impero" (Quarantotti Gambini 1971, 534). Considerato un traditore dagli austriaci e dal suo stesso padre, per gli italiani invece era considerato un eroe. Lo stesso padre di Paolo lo conosceva e gli aveva fatto vedere una sua foto:

Un pomeriggio durante la guerra, in casa a Trieste, il papà /.../gli aveva fatto vedere una fotografia su una rivista. Era, dal busto in su, un uomo dalla giubba scura abbottonata sino al collo, che aveva il naso un po' adun- co sul viso tondo, quasi grasso. "Guardalo bene, e non dire nulla a nessuno, - gli sussurrò il papà. - È un nostro ufficiale di marina che gli austriaci hanno impiccato. Era amico di Manlio e anche mio. Siamo stati a scuola insieme (Quarantotti Gambini I971, 535-536).

La confusione e il turbamento di Paolo raggiungono i picchi più alti nei passi finali del brano, quando ascolta i discorsi tra Matteo, legionario di Fiume, e il nonno. Matteo esprime la sua insoddisfazione per

$$
\begin{aligned}
& \text { /.../ quest'Italia convulsa, egoista, incoscien- } \\
& \text { te, che sembra nata non dalla vittoria ma dal } \\
& \text { dissolvimento di Caporetto, che noi tut- } \\
& \text { ti abbiamo combattuto? È per quest'Italia } \\
& \text { inetta, che non sa neanche governare i paesi } \\
& \text { liberati/.../ (Quarantotti Gambini I97I, 560) }
\end{aligned}
$$

e per la quale non si è raggiunto nulla. Il nonno di Paolo però replica:

- Nella mia vita ho veduto molte cose. Ero
bambino quando assistetti, nel I848, alla pri-
ma dimostrazione. Tutta una folla attraver-
sava la città /.../ gridando "Viva Pio IX, Viva
la Costituzione!", e quella sera vidi mio padre
rientrare con gli occhi sfavillanti e con la fa-
scia della Guardia Nazionale.../.../ - Da quel
giorno/.../ sono trascorsi più di settant'anni,
ein questi settant'anni c'è dentro tutto il nostro Risorgimento. Ebbene, credete che noi, ai nostri tempi, non abbiamo avuto le nostre delusioni? Ci sono stati momenti (Quando Garibaldi rispondeva "Obbedisco", quando Lamarmora, presidente dei ministri parlava di Trieste come di una città germanica, e quando re Umberto indossava la divisa bianca di colonnello austriaco per andar a stringere la mano a Francesco Giuseppe) che ci siamo sentiti perduti, poveri illusi beffati, e non abbiamo creduto più a nulla e a nessuno /.../. E ci sono stati altri momenti che ci siamo sentiti come te, Matteo; e avremmo voluto forzare le cose, sconvolgere il mondo: e fu in uno di questi momenti che Oberdan, che tra noi era il più giovane, appena un ragazzo, agì per tutti; e fu il primo dei nostri a 
conoscere la forca, quasi quarant anni prima

di Giusi" (Quarantotti Gambini 1971, 561).

Il pregiudizio di matrice nazionale che traspare da queste opere letterarie, si intreccia in alcuni casi con il pregiudizio di classe. Infatti Paolo, nonostante sia solo un bambino, prova quasi ribrezzo per coloro che non appartengono a una famiglia agiata come la sua. Durante l'incontro con una giovane coppia di contadini che il nonno aveva fatto salire in carrozza Paolo

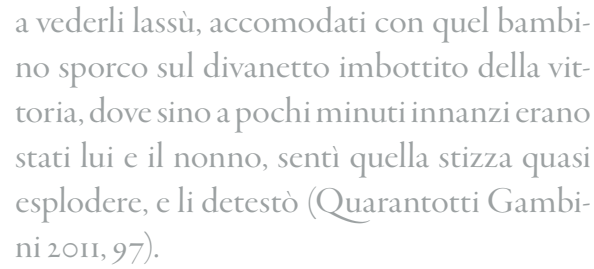

In questa prospettiva si inserisce anche l'incontro con il mondo slavo. Paolo continua a chiedersi: "Ma questi slavi, o schiavi, o s'ciavi, si può sapere chi sono?” (Quarantotti Gambini 1964, 51; 1969, 126). In un passo de Il cavallo Tripoli possiamo leggere:

\section{Slave erano le lavandaie e le donne che porta- vano latte e uova in città: le juzke dal fazzo- letto colorato in testa e dalle gonne lunghe larghe e spesse sotto il corpetto aderente che pareva un giacchettino da uomo, com'erano da uomo le loro scarpe quando non portava- no babbucce. Slave, anzi s'cave, erano le con- tadine che scendevano dai monti con fagot- ti, con cesti e coi vasi argentei azzurrini del latte (Quarantotti Gambini 1963, 106).}

L'elemento slavo in questi romanzi di Pier Antonio Quarantotti Gambini è subalterno, infatti slavi sono i coloni che lavorano nei campi o prestano servizio nella casa dei signori (Magris 20I8, XII). Da semplice differenza di classe sociale, si arriva però a un vero e proprio pregiudizio nazionale nei confronti degli slavi, esposto in Primavera a Trieste. Ricordi del '45, testimonianza in forma di diario in cui l'autore descrive i quaranta giorni d'occupazione di Trieste da parte dell'esercito jugoslavo nel 1945.
Le immagini dei soldati jugoslavi appaiono contrapposte a quelle dei soldati neozelandesi:
...poveri diavoli stremati, abbattuti, terri
bilmente straccioni in confronto coi neo- zelandesi dalle belle divise soltanto impol- verate, rosei, ben nutriti e ben sbarbati.
L'aspetto pietoso degli jugoslavi /.../ i loro
abiti scompagnati e i loro volti macilenti e sporchi di barba non rasa, non fanno che ac crescere, semmai, il disdegno dei triestini. (Quarantotti Gambini 20I8, II6).

In alcuni passi del libro, le considerazioni dell'autore sugli slavi si rifanno invece alle descrizioni dell'aspetto fisico:

Sono piccoli, in genere, questi sloveni; no tevolmente più bassi di quella che è la statura media dei triestini e degli istriani. Questi sloveni della campagna /.../ sembrano non cresciuti qui vicino ma di tutt'altri paesi, a paragone dei triestini che sono alti e baldi /.../. Le slovene, di corporatura corta e muscolosa /.../ sono esattamente l'opposto delle triestine, dai torsi slanciati e dalle gambe lunghe (Quarantotti Gambini 2018, I66).

La complessità del sentimento di apparenza nazionale però è interessato da un'evoluzione, infatti il bambino Paolo nei diversi romanzi inizia a porsi delle domande che potremmo definire tipiche delle rappresentazioni di un' identità “ $\mathrm{Alu-}$ ida" e in divenire.

Ne L'amore di Lupo, romanzo che narra le vicende al termine della guerra e descrive la vita di alcuni soldati italiani che si erano insediati nei pressi di Semedella, i reali caratteri degli italiani appaiono agli occhi del bambino per quello che sono. Se prima per anni aveva sentito parlare della loro gentilezza, della loro lealtà e del loro coraggio, ora che ha la possibilità di frequentarli e di conoscerli da vicino, Paolo può rendersi conto dei loro difetti. Dopo le vicende di Nerina, una ragazzina uccisa perché si era opposta alla violenza del soldato Frangisacchi (denominato Lupo per la sua meschinità), Paolo si chiede: 
Sono dunque così gli italiani? Sono tutti

cosi, e non gentili, coraggiosi e leali, come

diceva un tempo la mamma? (Quarantotti

Gambini 1964, 192).

Frangisacchi si prende gioco anche dei sentimenti irredentisti della famiglia di Paolo:

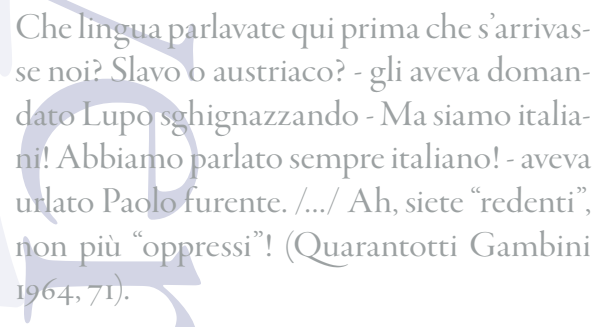

Ma i ripensamenti di Quarantotti Gambini non sono solo nei confronti degli stessi soldati italiani, bensì anche degli slavi. In Primavera a Trieste, c'è un passo importante in cui si racconta di un fatto che lo scrittore ha vissuto quando si trovava a Milano come studente. In tram ha sentito i commenti della gente contro un gruppo di agitatori slavi della Venezia Giulia che hanno provocato agitazione nel giovane:

pensando in quel momento agli slavi dell'I stria, li sentii, come non mi era mai avvenuto, gente della mia terra, istriani come me, anche se d'altra stirpe. L'essere nati e cresciuti in uno stesso paese, sotto lo stesso sole, davanti allo stesso mare, non imprime un carattere comune? Edunque potevo sentirmi vicino agli slavi della mia terra; ed essi potevano in qualche modo sentirsi più vicini a noi italiani dell' Istria”. E prosegue: "non sentii più nessuna differenza tra noi e gli slavi; essi erano come noi, e noi come loro: istria ni (Quarantotti Gambini 20I8,331).

È significativo in conclusione vedere come nell' introduzione a Primavera a Trieste Quarantotti Gambini abbiamo ricordato che:

questo libro (nato dalla fede nella capaci tà dello spirito di sanare la ferite aperte dalla realtà) non vuole essere un atto d'accusa per nessuno; ma indicare semmai, attraverso il travaglio umano di chi è la testimonianza, quali sono le cose che rifiutiamo, per noi e per gli altri: per chi potrebbe subirle domani, come per chi le ha subite ieri per nostra colpa. E come noi le rifiutino, nel passato e nell'avvenire, quanti vogliono credere che valga ancora la pena di essere uomini (Quarantotti Gambini 20I8, 45).

\section{Conclusione}

Nel presente contributo, partendo dalle impostazioni teoriche dell'imagologia letteraria, è stata condotta un'analisi critica di alcune opere narrative di Pier Antonio Quarantotti Gambini relativa alle immagini letterarie veicolate dal testo, le cosiddette auto-rappresentazioni (auto-images) e le immagini dell'“altro" (hetero-images). Nelle opere che sono state prese in considerazione, in ragione soprattutto della loro pertinenza con la tematica dell' identità e dell'alterità, si può notare come per lo scrittore, e per estensione per gli italiani d'Istria, la lingua è un forte elemento costitutivo dell'appartenenza nazionale. Nei periodi di instabilità politica e di grandi sconvolgimenti storici come quelli descritti da Quarantotti Gambini nei romanzi che compongono la raccolta Gli anni ciechi, si può individuare una sequenza ricorrente: auto-rappresentazioni positive per le descrizioni degli italiani ed etero-rappresentazioni negative per descrivere le caratteristiche degli austriaci e degli slavi.

Paolo, personaggio chiave del "ciclo" ed alter ego dello scrittore, rappresenta tuttavia un caso particolare, perché costituisce un caso di un'identità in divenire. Nonostante lo scrittore, e di conseguenza la famiglia Amidei Brioresi, siano animati da una forte coscienza nazionale italiana, con il passare del tempo trova spazio la riflessione sulla condizione dei diversi gruppi etnici e/o nazionali. Sebbene in Quarantotti Gambini sia forte il sentimento nazionale e della patria perduta, ciò che conta è non dimenticare il passato e ricordarsi dell'elemento umano.

\section{Povzetek}

Namen članka je bilo poglobiti temo nacionalne identitete in njenega trka z "drugim" z uporabo imagološke perspektive v pripovednih delih Pier Antonia Gambini- 
ja. Avtorjevo življenje se je prepletalo z velikimi političnimi pretresi prve polovice dvajsetega stoletja. Rodil se je leta I9ro v istrskem Pazinu, v času Habsburške monarhije, leta 1918 je bil priča pripojitvi ozemlja Kraljevini Italiji, mnogo let pozneje, leta 1945, pa še okupaciji Trsta s strani Titove vojske, po kateri je oblast v Istri, Kvarnerju in Dalmaciji prevzela Jugoslavija.

V delih, izbranih predvsem zaradi tematike identitete in drugačnosti, je razvidno, da predstavlja jezik tako pisatelju kot ostalim istrskim Italijanom močan element nacionalne pripadnosti. V obdobjih politične nestabilnosti in velikih zgodovinskih preobratov, kot jih je opisal Quarantotti Gambini v romanih, ki sestavljajo zbirko Gli anni ciechi, je mogoče prepoznati ponavljajoče se zaporedje: pozitivne opise Italijanov in negativno opisovanje značilnosti Avstrijcev in Slovanov.

Čeprav so v delih Quarantottija Gambinija prisotna nacionalna čustva in hrepenenje po izgubljeni domovini, si ne smemo dovoliti, da bi pozabili na preteklost in človeški element v njem.

\section{Summary}

In the present article the author analyzes the theme of national identity and its comparison with the Other in the narrative works of Pier Antonio Quarantotti Gambini, using the imagological approach. Pier Antonio Quarantotti Gambini, a writer from an aristocratic family of irredentist ideals, has always highlighted the Italianness of Istria and Venezia Giulia. He lived during periods of political instability and tumultuous years of the first half of zoth century. Born in Pisino d'Istria at the time of Austria-Hungary, in 1918 he witnessed the Empire dissolution and the annexation to Italy, then later in 1945 he witnessed the occupation of Trieste by $\mathrm{Ti}$ to's troops and the passage of Istria and Dalmatia to the territories of Yugoslavia.

In his narrative works, especially in the novels of the collection Gli anni ciechi, the Italian national identity is expressed especially through language and culture. In those literary works a recurrent sequence can be identified: positive self-representations are used for the descriptions of the Italians and negative hetero-representations are used to describe the characteristics of the Austrians soldiers and the Slavs. However, although the national feeling is strong in Quarantotti Gambini narrative, what matters for the author is remembering the past and the people.

\section{Riferimenti bibliografici}

Beller, M., e J. Leerseen. 2007. Imagology. The cultural construction and literary representation of national characters. Amsterdam-New York: Rodopi.

Gallo, C. 2012. "Il mito dell'italianità in uno scrittore di confine: Pier Antonio Quarantotti Gambini”. In La letteratura degli Italiani 3. Gli Italiani nella letteratura. Atti del XV Congresso Nazionale dell'Associazione degli Italianisti Italiani (Torino, I4-17 settembre 2011), a cura di Clara Allasia, Mariarosa Masoero e $\downarrow$ Laura Nay, 1545-1555. Alessandria: Edizioni dell'Orso.

Iannuzzi, G. 2016. "Dizionario biografico: Quarantotti Gambini, Pier Antonio”. Treccani.it: http://www.treccani.it/ enciclopedia/quarantotti-gambini-pierantonio_(Dizionario-Biografico).

Maculotti, M. 2016. "Le redini e la corsa. Una lettura critiche degli Anni ciechi di Pier Antonio Quarantotti Gambini”. Tesi di laurea magistrale, Università degli Studi di Milano, Facoltà di studi umanistici.

Maier, B. 1996. La letteratura italiana dell'Istria dalle origini al Novecento. Trieste: Edizioni Italo Svevo.

Moll, N. 1999. "Immagini dell'“altro". Imagologia e studi interculturali”. In Introduzione alla letteratura comparata, a cura di Armando Gnisci, 21 I-249. Milano: Bruno Mondadori.

Moll, N. 2018. "L'imagologia interculturale nell'attuale contesto culturale e mediale". In Interpretare l'immagine letteraria dell'alteritá. Prospettive teoriche e critiche comparate, a cura di Franca Sinopoli e Nora Moll, 157-I77. Roma: Lithos.

Moretto, M., e D. Picamus. 2010. Quarantotti Gambini: l'onda del narratore. Trieste: Comune di Trieste. 
Quarantotti Gambini, P. A. 1963. Il cavallo Tripoli. Torino: Einaudi.

Quarantotti Gambini, P. A.. 1964. L'amore di Lupo. Torino: Einaudi.

Quarantotti Gambini, P. A. 1967. La rosa rossa. Milano: Mondadori.

Quarantotti Gambini, P. A. 1969. La corsa di Falco. Torino: Einaudi.

Quarantotti Gambini, P. A. 1971. Gli anni ciechi. Torino: Einaudi.

Quarantotti Gambini, P. A. 2or. Le redini bianche. Milano. Isbn Edizioni.

Quarantotti Gambini, P. A. 2018. Primavera a Trieste. Ricordi del '45. Milano: Mondadori.

Tremul, M., a cura di. 2008. Pier Antonio Quarantotti Gambini. Atti del Convegno di studi (Capodistria, 22 febbraio 2008). Capodistria: Unione Italiana.

Zudič Antonič, N. 2008. "Immagini capodistriane tra il ricordo dello scrittore e il presente". In Pier Antonio Quarantotti Gambini. Atti del Convegno di studi (Capodistria, 22 febbraio 2008), a cura di Maurizio Tremul, 4I-45. Capodistria: Unione Italiana.

Zudič Antonič, N. 20I 4. Storia e antologia della letteratura di Capodistria, Isola e Pirano. Capodistria: Unione Italiana.

Zudič Antonič, N. 2018. "Rappresentazione dello spazio narrativo capodistriano attraverso i romanzi di Pier Antonio Quarantotti Gambini”. In La città italiana come spazio letterario nel contesto mediterraneo (I9go-2015), a cura di Srećko Jurišić, Antonela Marić, Nikica Mihaljević e Katarina Dalmatin, II9-I27. Firenze: Franco Cesati Editore. 